\title{
Designing Notifications for Ubiquitous Monitoring Systems
}

\author{
Stuart Moran and Joel E. Fischer \\ Mixed Reality Lab \\ University of Nottingham \\ Nottingham, UK \\ firstname.lastname@nottingham.ac.uk
}

\begin{abstract}
Pervasive computing systems involve a number of different embedded, mobile and sensory devices. To provide their intended service or application, these devices will collect unprecedented levels of data about users as they go about their daily lives. Users are anticipated to seamlessly, and frequently, physically move between numerous monitoring systems on a regular basis. As a result, it may become difficult for users to comprehend and keep track of the systems that monitor them, how their data is used, and to give informed consent. One solution is to use smart phones to selectively notify users to changes in the monitoring systems around them. However, there is often a problem in identifying and designing for the opportune moment to send a notification. This paper describes an application of the Contextual Factors of Interruption (CFI) model in designing three different types of notifications useful to users of ubiquitous monitoring systems.
\end{abstract}

Keywords- Notifications, Modelling, Monitoring, Ubiquitous

\section{INTRODUCTION}

A few decades from now, we will see computing systems that literally pervade almost all aspects of our lives. These pervasive systems will consist of substantial numbers of connected embedded, mobile and sensory devices. These devices will collect unprecedented levels of information about our actions, preferences and environments; a process termed Ubiquitous Monitoring (UM) [1]. The primary purpose of these widespread systems is to provide a new means of interacting with computers, which enable highly novel applications and services designed to improve our lives. Viewing our interaction with computers as a simple, and limited, one-to-one relationship will no longer be appropriate, as in the future they will function in a more fluid and dynamic way around us. This is akin to the vision of Weiser [2], which is being realized through ongoing developments in Ubiquitous Computing. There are countless envisioned benefits to these systems, ranging from more efficient management and care of patients in hospitals [3] to ensuring the safety of children in amusement parks [4]. With so many potential areas of benefit, there will likely be a significant number of different monitoring systems, covering almost every aspect of our daily lives.

An interesting problem to consider is the means by which users might consent to participation in such systems. Consent is often sought implicitly in existing systems such as CCTV, likely due to their simple, passive and wellunderstood functionality and purpose. However, with respect to future systems, which are expected to be considerably more dynamic and complex, a more proactive and explicit request for consent will likely need to be made. When this is considered alongside the anticipated large numbers of monitoring systems, collecting different types of data, for different purposes, the cognitive demands on users are likely to increase significantly. Hence it will likely become difficult for users to keep track of, and manage, this information

Approaches already exist at raising awareness and seeking consent. For example, signs are displayed on walls are used to inform users that CCTV is in operation [5]. With regards to consent, it is common to agree to terms and conditions prior to usage, by signing a document or simply clicking a check box. Unfortunately, these approaches are unlikely to be feasible for future monitoring systems. In terms of signage, there are likely to be too many systems collecting a variety of different data types to capture in a simple sign. With respect to consent, given the highly dynamic nature, a single point or moment of consent will no longer be sufficient; particularly given the systems will be embedded and hidden from view, and constantly changing. Hence, a key question is how can users of future UM systems be appropriately (and unobtrusively) be made aware of, informed about, and give consent to, their use?

Given the current proliferation of smart phones, it is reasonable to assume that the majority of people will have access to one in the future. As such, one effective solution to the problem of data management and consent in UM systems might be to utilize smart phone-based notifications. Such notifications could be sent to users as they move between monitoring systems, updating them on any recent changes to the system at opportune and appropriate moments. They could also be used to actively seek consent for participation, activate certain functions or applications, and inform recipients of the data types being collected. While notifications can be utilized to deliver important information, they may potentially interrupt an individual's activity in a disruptive way. In some cases it may even endanger people's lives if the notification is received at the wrong time, for example in complex multi-tasking environments such as driving [6]. This highlights one of the predominant difficulties of designing notifications: people's receptivity varies moment-by-moment. The Contextual Factors of Interruption (CFI) model was developed in light 
of this problem, which can help analyze the various properties of a notification as well as the user's context, helping identify likely key moments of high-receptivity [7].

The aim of this paper is to explore the use of this model as a means of designing notifications suitable for informing people, and requesting their consent, as they move between UM systems. We use an envisioned future scenario to help highlight the issues of monitoring systems and notifying users, and design three types of monitoring-based notifications using the model.

\section{UBIQUITOUS MONITORING}

As technology becomes more connected, mobile and embedded, we will see the emergence of a more cohesive and pervasive range of monitoring systems. Ubiquitous Monitoring (UM) is defined as the use of pervasive devices for continuous collection of data on a large scale, based on [1]. Many of the benefits of pervasive computing are centered on the ability to collect a large corpus of data about users, making UM a fundamental process to many applications. The potential benefits of this degree of monitoring are numerous, particularly when environments such as hospitals, schools, offices, homes, shopping centers and even vehicles begin to share data. As mentioned earlier, these systems will be so numerous that people are likely to pass between different systems several times during a single day. These systems will be designed for different purposes, collect different types of data, have different owners and request different terms for participation. To help highlight the implications of some of the application areas, and potential issues involving these systems, an envisioned example far-future scenario describing a day in the life of 'John' is presented; analogous to Weiser's 'Sal' [2]. The scenario is designed around a number of themes (based on [1]) central to users interaction with UM systems, including:

- Public \& Private Spheres: as monitoring systems increase, people will move seamlessly between them, and the line between public and private will blur.

- Situation \& Context: future monitoring systems are expected to exist in a number of different contexts such as the home, transport, security, healthcare and more.

- Data Types \& Justification: a variety of data on user preferences, behaviors and biometrics will be collected for security, health and personal gain.

- Autonomy \& Agency: software agents will assist users in the management of information collected about them, and control (and act as a gateway to) many applications.

The aim is to create a representative vision of UM systems that are grounded in existing monitoring prototypes, principles, and practices. These existing technologies ${ }^{1-7}$ are used to inform and build our projections of future interactions and applications. It should be noted that there are a number of notifications described that enable the systems John experiences to interact with him. These differ from the notifications being explored in this paper, which are about the system (i.e. meta), and are intended to support users as they move between these monitoring systems.

\section{A. Example Future Scenario}

The intelligent home management system (IHMS) installed in John's house projects a message on to the wall to remind him that he needs to pick up Mary from the hospital today. John picks up his car keys, triggering a transdermal sensor that scans his sweat and informs him that there is still alcohol in his body from the club last night, and that he should not drive [c.f. ${ }^{1}$. The IHMS then informs John of the most convenient bus route to the hospital, and prepurchases and prints him a ticket. As John makes his way out of the house, the IHMS automatically locks the windows and doors, and starts to make preparations for dinner based on John and Mary's projected arrival back home [c.f. $\left.{ }^{2}\right]$.

Walking down the street toward the bus stop, a government owned display screen makes an inference about John's intention to take the bus, and informs him that a traffic accident has just occurred nearby which will affect all bus times. It then suggests to John that if he shares his intended destination, it will calculate a more efficient bus route for him $\left[c . f^{3}\right]$. John obliges, and is guided to a new bus stop ten minutes walk away. When the bus arrives, John gets on and inserts his ticket into the collector. The onboard system reacts by welcoming John by name, and then guides him to a window seat based on his preferences profile with the transport company $\left[c . f^{4}\right]$. Along the way, a series of advertising promotions are directed at John, related to the club he attended yesterday. This information was gathered from the taxi he ordered last night from the club, which happens to be owned by the same transport company [c.f.5]. As John is quite tired, he falls asleep on the bus. Luckily for him a small vibration in the seat wakes him as the bus arrives at the hospital. As he gets off the bus, he sees an advertisement on the bus window for a coffee shop just around the corner; he decides to get a drink to perk himself up. Walking toward the hospital entrance, John takes a shortcut through the car park. The local security system picks up on John's actions, and notes that he did not arrive in a car or recently leave from the hospital [c.f. ${ }^{6}$. The system then treats John with suspicion, and does a cross check with the local police database. With nothing of significance on record, the system announces to John that it would be safer for him to stick to the pavement. Surprised, John quickly returns to the pavement and the system thanks him for his co-operation.

As John arrives at the hospital, the intelligent entrance system requests that he provide a sample of blood before entering, which he politely declines. The system then

\footnotetext{
${ }^{1}$ http://tinyurl.com/9x4d4tz, ${ }^{2}$ http://tinyurl.com/yaxub9h,

${ }^{3} \mathrm{http}: / /$ tinyurl.com/957svk9, ${ }^{4} \mathrm{http}: / /$ tinyurl.com/yyh9mgd,

${ }^{5} \mathrm{http}: / /$ tinyurl.com/9lc9plc ${ }^{6} \mathrm{http} / /$ tinyurl.com/6xwjxrr

${ }^{7}$ http://tinyurl.com/9uu4yx3
} 
informs John that he will not be permitted to enter the hospital unless he is checked for potential contagious viruses. Reluctantly he gives a sample of his blood, and is permitted entrance with a polite suggestion that he try to keep the number of units of alcohol he drinks down. A screen on the wall asks John a series of questions to confirm his relation to Mary, after which he is automatically guided through the hospital by projections on the walls [c.f.].

This example scenario highlights a number of interesting aspects of a variety of UM systems. Firstly, when John is at home he has permitted the system to act autonomously on his behalf. He has also given the system access to personal data such as daily schedules and movement patterns. He owns this system, and hence has complete control. When walking to the bus stop, he is anonymously tracked by the public service display screen, and is requested to share his destination data. On the bus, we see that John has a prestated agreement with the transport company. In exchange for information regarding his transport behaviors, he gains discounts and a personal preferences profile. This allows the company to display targeted advertisements at John. In addition, we see that the taxi service John used is linked to his profile with the transport company, which shares data across its systems. As is commonplace in today's society, security measures (in the form of cameras) are present in most places of risk. In the car park, John was not aware that the security system was monitoring him; the fact that he simply entered the space was viewed as consent to both participation in the monitoring system and the crosschecking of data. At the hospital, John had to give some of his blood to be tested; this could be viewed as justified. He had no choice but to sacrifice this information to make use of/gain access to the service.

The above scenario helps envisage a future of monitoring systems with complex issues related to permissions, control, consent, autonomy, personal data types, awareness, ownership, and public and private spaces; these are just some of the many factors to consider when designing monitoring systems [1]. Notice that in the scenario, John was not notified of the systems he was using, or any 'recent' changes to the data or terms of participation. One of the problems for the future is keeping track of personal data, enacting upon different levels of control, and giving consent as people move through these constantly updating systems. As such, we propose smart phone based notifications as one solution.

\section{NOTIFICATIONS AND INTERRUPTIONS}

Cognitive psychology has suggested that an interruption is "an externally generated randomly occurring, discrete event that breaks continuity of cognitive focus on a primary task" [8]. Thus, in its definition it describes a cognitive phenomenon that occurs to the recipient of an interruption. In this paper, a notification can be understood as an explicitly designed, technology-mediated interruption, with the intent to deliver information to the recipient. It is a kind of message designed as an announcement to gain the recipient's attention on its delivery.

Notifications play a key role in the design of user interfaces in general and mobile devices in particular. They announce incoming communication such as email, voice calls, text messages, and events in social networking sites such as when someone has tagged us on a photo or mentioned us in a tweet. They also announce system updates and prompt us to confirm whether we really want to close an application. There may be a complex tension between the perceived benefits of a notification and the potential disruptiveness an interruption may cause [9].

Beyond notifications, studies of interruption have shown that they are a frequent method to initiate conversations [10] and information exchange [11] in the workplace. Another study reveals that healthcare professionals are routinely interrupted throughout their day, which they reported leads to errors in using a patient care information system [12]. The case illustrates how interruptions are a routine part of some settings, and how contextually ill-designed information systems may be hazardous. What are the contextual circumstances that characterize the positive or negative experience or effects of an interruption?

\section{CONTEXTUAL FACTORS OF INTERRUPTIONS}

The Model of Contextual Factors of Interruptions (CFI) (see Figure 1) was developed by Fischer [7] and is grounded in findings from the literature on interruption studies. In this paper, we use the CFI model to inform the design of monitoring based notifications so as to improve their acceptability. Other models exist that can assist in designing interruptions, such as McFarlane's taxonomy [19], but (unlike the CFI) fails to consider the contextual factors in the immediate environment of the recipient and their ongoing social involvement with other people.

The CFI model identifies and relates the main entities involved in the interruption process, which act as the antecedents of notification receptivity [7]. These entities are categorized into two types of contextual factors: local e.g. the environment of the recipient and relational e.g. the content of the interruption, both of which are discussed in detail in the following sections [7].

\section{A. Local Contextual Factors}

These factors account for the local context of the recipient of a notification, and include: embodied, environment and social factors. Embodied factors account for a recipient's involvement in the world including their cognitive and physical activities, mental state, emotions, intentions and collected experiences [7]. Environmental factors capture the appearance, configuration and appropriation of the space surrounding the recipient, including the social, structural and physical space. This is an important consideration, as one is likely to be more receptive to an interruption on a bus than in a church. Social factors capture the presence, role, 
relationship and engagement of other people with the recipient [7]. For example, consider a recipient riding on a bus. The local contextual factors can be used to effectively capture their current situation. The bus is a moving vehicle, with seats in rows with the primary purpose of transporting people (environmental). The recipient is sitting on their own but with a number of people around them (social), and they are currently engaged with an amusing book (embodied). This information can be used to inform the design of appropriate notifications, which can be described by the relational contextual factors.

\section{B. Relational Context Factors}

Relational contextual factors focus on aspects of the interruption itself, and include: the sender, content, medium, timing and presentation of the interruption. Fischer develops these by referencing a wide range of empirical studies of interruptions. For example, the identity of a notifications sender has been shown to be an important consideration for the recipient's receptivity and responsiveness [13], [14]. Further, a number of empirical studies have found that if the content of the notification is perceived as urgent [15], relevant [16], and/or complex [17] responsiveness can change. This can also be influenced by the way an interruption is presented, for example if the notification is delivered directly to the screen (an immediate interruption), or if the user needs to accept the notification first (a negotiated interruption) [18].

Interruptions' can be conveyed through different media. In addition to more traditional channels such as mobile phones and personal computers, some interesting work has also explored the effects of heat, light, odour, vibration and sound of interruptions on responsiveness [19], [20]. Finally, when identifying opportune moments for notifications, timing is a core consideration [21]. Studies have shown that receptivity is lower for interruptions when they occur just before a new task [22], or during a cognitively demanding task [23]. Returning to our bus example, we can capture the properties of an interruption using the factors outlined above. A recipient is receiving an interruption from their mother (sender) regarding the well being of their father (content); which they were expecting. The interruption was in the form of a phone call (medium) and was unnoticed by other people because the alert was through vibration (presentation). The call was received when the recipient was not engaged in any other important activity, making it an opportune moment (timing).

\section{NOTIFICATIONS AND UM SYSTEMS}

Using the CFI model, we present three different notifications that have been designed to help John (from the example scenario) understand and give consent to the monitoring systems around him. Knowledge of the local context is required for these notifications to be successfully received. By coincidence, the future monitoring systems being discussed will likely already capture this information effectively as a part of the services they provide. As such, this information can be assumed to be known, and hence can inform the properties of the notification being designed.

In Table 1 three monitoring systems have been identified from the future scenario. The first describes the situation where John was riding the bus. The interruption that has been designed for this situation is referred to as an 'informative interruption'. The content of the interruption informs John that there is a change in the terms of service regarding the data being collected by the transport company. As John is asleep on the bus, the timing of the notification is delayed until he is awake (as there is little urgency in the content). In addition, the interruption is presented in the form of a negotiation, so John is not overwhelmed upon waking and can receive the notification in his own time.

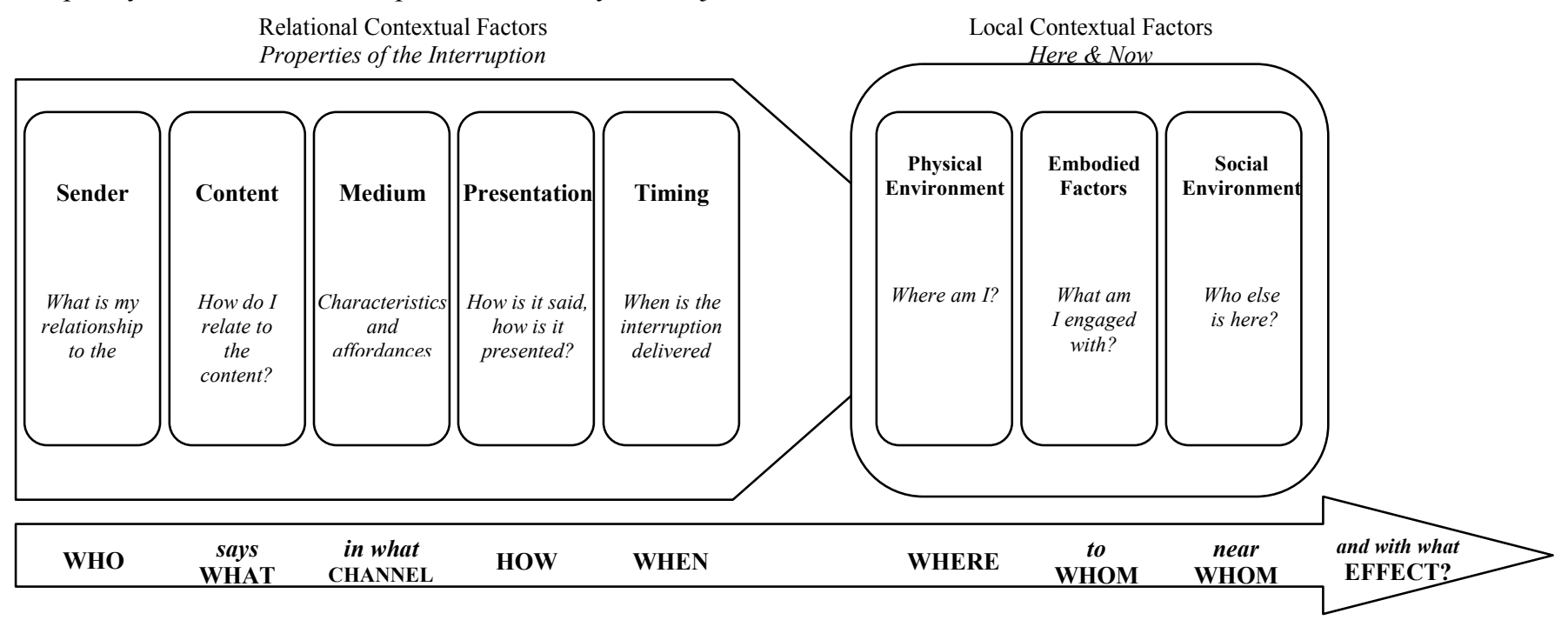

Figure 1. Contextual Factors of Interruptions (CFI) Model, based on [7] 
The second situation is where John was walking through the car park, unaware that he was being observed. The interruption designed for this scenario is referred to as an 'awareness interruption'. It has been designed to alert John to the fact that he has recently entered an area that is being monitored. The interruption informs John of the purpose of the system, the types of data being collected and that entering the space is considered consent to participation. The interruption is relatively urgent, as John should be provided with this information prior to entering the space. As such the interruption is immediate, and is in the medium of both vibration and sound, to capture John's attention.

The third and final monitoring system is the entrance to the hospital. The interruption for this scenario is referred to as a 'consenting interruption'. Given that John must consent to his blood being taken, he should be informed about the purpose, storage and use of this data, including knowledge of other monitoring systems within the hospital. This should be done with sufficient time for him to consider his options, to which he can then give his consent via the notification. This is an interruption that takes precedence in John's phone, as it requires a response, and given its importance, he is alerted to it by both vibration and sound. The timing of the interruption would be during his walk toward the hospital, as he is not engaged with an activity.

With the potential for many types of interruption, it is important to consider how they might be prioritized. Consenting interruptions will likely take top priority, given the potential implications of the data use. Second to which are awareness interruptions which are time critical and only have value prior to entering a system, followed by, informative interruptions. The format, content and length of the interruption is also likely to vary depending on the type e.g. an awareness interruption might simply be a symbol, while a consenting interruption will likely contain considerable text.
The CFI model has been used to design notifications for future monitoring systems, but it can also be used on existing ones. For example, consider a newly installed CCTV camera on a shopping high street. A person might be walking down the street (environment), with a friend (social), looking through the windows of shops at items of clothing (embodied). Given this representation of the local contextual factors, we can now consider the role of the properties of our interruption in determining receptivity. The sender of the interruption would be the owner of the camera, with content describing the fact that camera is new, its purpose and its position. Given that the recipient is engaged in window-shopping, the notification should be presented in text format, and the medium should be an alert in the form of a vibration (so as to not distract). The timing of the notification should be while the recipient is within range of the camera, so they can refer to its position when reading the notification. Whilst content and sender are predetermined by intent of the notification, taking the local contextual factors into account in the design choices of presentation, medium and timing of the notification will likely increase notification acceptance rate and impact.

Using Fischer's [7] model, a series of notifications have been designed for different ubiquitous (and existing) monitoring systems. Using the properties of a notification it is possible to design notifications which better coincide with a recipient's local context, potentially increasing their level of responsiveness. While the notifications may not be the most appropriate or realistic for each situation, they do highlight the different ways in which notifications can be designed and used in monitoring systems: ranging from receiving information, to giving it. It is important that recipients receive this information in situ as it may help them better appreciate the implications of the monitoring systems they are a part of, and reinforce the knowledge that they are being observed.

Table 1 A description of local contextual factors and properties of interuption in three envisoned monitoring systems

\begin{tabular}{|c|c|c|c|c|}
\hline & \multicolumn{3}{|c|}{ Designed Interruptions } \\
\hline & & $\begin{array}{c}\text { Riding the Bus } \\
\text { (Informative Interruption) }\end{array}$ & $\begin{array}{l}\text { Walk through Car Park } \\
\text { (Awareness Interruption) }\end{array}$ & $\begin{array}{l}\text { Approaching Hospital } \\
\text { (Consent Interruption) }\end{array}$ \\
\hline \multirow{3}{*}{ 胥 } & Environment & $\begin{array}{l}\text { Moving space with rows of chairs } \\
\text { designed to transport people }\end{array}$ & Space designated for parking cars & Space designed for treating people \\
\hline & Social & A number of passengers are present & There is nobody else present & There are passersby present \\
\hline & Embodied & Recipient is asleep & $\begin{array}{l}\text { Recipient is distracted by task of } \\
\text { getting to hospital }\end{array}$ & Recipient's desire is to enter hospital \\
\hline \multirow{5}{*}{ 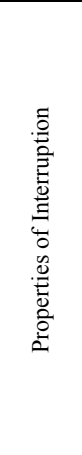 } & Sender & Owner of company & Owner of car park & Hospital Manager \\
\hline & Content & $\begin{array}{l}\text { Information regarding impending } \\
\text { changes in terms of service and price } \\
\text { plans }\end{array}$ & $\begin{array}{l}\text { Inform recipient that there is a } \\
\text { monitoring system in place, its } \\
\text { purpose and that crosschecks are } \\
\text { made with government documents. } \\
\text { Also explain to recipient the need for } \\
\text { this data, and how it is used }\end{array}$ & $\begin{array}{l}\text { Request that recipient give consent to } \\
\text { have their blood tested, prior to entrance } \\
\text { to hospital. Information about being } \\
\text { tracked around hospital. Also explains to } \\
\text { recipient the need for this data, and how } \\
\text { it is used }\end{array}$ \\
\hline & Presentation & $\begin{array}{l}\text { Negotiated text based interruption - } \\
\text { non-essential content, should be read }\end{array}$ & $\begin{array}{l}\text { Immediate text based interruption - } \\
\text { given urgency and security intent }\end{array}$ & $\begin{array}{l}\text { Immediate text based interruption } \\
\text { given requirement for consent for access }\end{array}$ \\
\hline & Medium & $\begin{array}{l}\text { Vibration only -not to disturb other } \\
\text { passengers }\end{array}$ & Vibration and sound - for urgency & Vibration and sound - for urgency \\
\hline & Timing & $\begin{array}{l}\text { Observe that recipient is asleep. Alert } \\
\text { to notification once they are awake and } \\
\text { not engaged in a task }\end{array}$ & $\begin{array}{l}\text { Infer intention of recipient to walk } \\
\text { through space, and interrupt them } \\
\text { prior to entrance. }\end{array}$ & $\begin{array}{l}\text { Observe recipients intention to enter } \\
\text { building, and interrupt prior to their } \\
\text { arrival to give time for understanding }\end{array}$ \\
\hline
\end{tabular}




\section{CONCLUSIONS AND FUTURE WORK}

In this paper we have presented an envisioned scenario of future ubiquitous monitoring systems. The intention was to highlight some of the complex issues and experiences that will likely occur as people move between systems. Using the CFI model, we designed a series of smartphone-based notifications intended to inform users, increase their awareness and seek their consent to participation. We were able to capture and represent the local contextual factors that affect users' receptivity, and use this to inform the design properties of the notification for each instance. The main contribution of this work is in highlighting some of the complexity in future monitoring systems and demonstrating one possible design solution to unobtrusively assist users as they traverse between them. However, this work is only a starting point from which to begin to study the experiences of people in UM systems and a more comprehensive framework is likely needed before it can be applied in practice.

The next steps for this research are to explore how users respond to monitoring-based notifications designed using the CFI model in the real world, using current systems such as CCTV and GPS. This will be run in parallel with studies looking at the approaches people currently use when dealing with multiple different types of monitoring system. Another direction is to theoretically augment Fischer's framework with aspects of the Technology Acceptance Model (TAM) [24] or the Perception of System Attributes - Behavioral Intention (PSA-BI) Model [25]. Here we could investigate whether a recipient's intention to accept a notification involving a monitoring system can be directly predicted. Such work could provide the foundation logic upon which notifications are automatically designed and distributed.

\section{REFERENCES}

[1] S. Moran and K. Nakata, "Analysing the Factors Affecting Users in Intelligent Pervasive Spaces," Intelligent Buildings International: Special Issue on Intelligent Pervasive Spaces for Working and Living, vol. 2, no. 1, pp. 57-71, 2010.

[2] M. Weiser, "The Computer for the 21st Century," Scientific American, vol. 265, no. 3 , pp. 94-104, 1991.

[3] S. Mitchell, M. D. Spiteri, J. Bates, and G. Coulouris, "ContextAware Multimedia Computing in the Intelligent Hospital," in Proceedings of the 9th workshop on ACM SIGOPS European workshop: beyond the PC: new challenges for the operating system, 2000, pp. 13-18.

[4] X. Lin, R. Lu, D. Kwan, and X. (Sherman) Shen, "REACT: An RFID-based privacy-preserving children tracking scheme for large amusement parks," Computer Networks, vol. 54, pp. 2744-2755, 2010.

[5] R. Lippert, "Signs of the Surveillant Assemblage: Privacy Regulation, Urban CCTV, and Governmentality," Social and Legal Studies, vol. 18 , no. 4, pp. 505-522, 2009.

[6] S. T. Iqbal, Y.-C. Ju, and E. Horvitz, "Cars, calls, and cognition: Investigating driving and divided attention," in Proceedings of the SIGCHI conference on Human factors in computing systems, CHI '10, 2010, pp. 1281-1290.

[7] J. E. Fischer, "Understanding Receptivity to Interruptions in Mobile Human-Computer Interaction," University of Nottingham, 2011.
[8] L. Coraggio, "Deleterious effects of intermittent interruptions on the task performance of knowledge workers: A laboratory investigation," University of Arizona, 1990.

[9] J. M. Hudson, J. Christensen, W. A. Kellogg, and T. Erickson, “'I'd Be Overwhelmed, But It's Just One More Thing to Do:' Availability and Interruption in Research Management," in In Proceedings of the SIGCHI conference on Human factors in computing systems, CHI '02,, 2002, pp. 97-104.

[10] S. Whittaker, D. Frohlich, and O. Daly-Jones, "Informal workplace communication: What is it like and how might we support it?," in Proceedings of the SIGCHI conference on Human factors in computing systems, CHI '94, 1994, pp. 131-137.

[11] B. O'Conaill and D. Frohlich, "Timespace in the workplace: Dealing with interruptions," in Proceedings of the SIGCHI conference on Human factors in computing systems, CHI '95, 1995, pp. 262-263.

[12] J. S. Ash, M. Berg, and E. Coiera, "Some unintended consequences of information technology in health care: The nature of patient care information system-related errors," Journal of the American Medical Informatics Association, vol. 11, no. 2, pp. 104-115, 2004.

[13] S. A. Grandhi and Q. Jones, "Conceptualizing interpersonal interruption management: A theoretical framework and research program," in Proceedings of 42nd International Conference on System Sciences, 2009, pp. 1-10.

[14] D. Avrahami, S. R. Fussell, and S. E. Hudson, "IM waiting: Timing and responsiveness in semi-synchronous communication," in Proceedings of the ACM conference on Computer supported cooperative work, CSCW '08, 2008, pp. 285-294.

[15] M. H. Vastenburg, D. V. Keyson, and H. de Ridder, "Interrupting people at home," in Proceedings of the 2004 IEEE conference on systems, man and cybernetics, 2004, pp. 59-64.

[16] S. T. Iqbal and B. P. Bailey, "Effects of intelligent notification management on users and their tasks," in Proceedings of the SIGCHI conference on Human factors in computing systems, CHI '08, 2008, pp. $93-102$.

[17] T. Gillie and D. Broadbent, "What makes interruptions disruptive? a study of length, similarity, and complexity," Psychological Research, vol. 50, pp. 243-250, 1989.

[18] D. C. McFarlane, "Comparison of four primary methods for coordinating the interruption of people in human-computer interaction.," Human-Computer Interaction, vol. 17, no. 1, pp. 63139, 2002.

[19] E. Arroyo and T. Selker, "Self-adaptive multimodal-interruption interfaces," in IUI '03: Proceedings of the 8th international conference on; Intelligent user interfaces, 2003, pp. 6-11.

[20] E. Arroyo, T. Selker, and A. Stouffs, "Interruptions as multimodal; outputs: Which are the less disruptive?," in ICMI '02: Proceedings; of the 4th IEEE International Conference on Multimodal Interfaces, 2002, p. 479.

[21] D. C. McFarlane and K. A. Latorella, "The scope and importance of human interruption in human-computer interaction design," HumanComputer Interaction, vol. 17, no. 1, pp. 1-61, 2002.

[22] C. A. Monk, D. A. Boehm-Davis, and J. G. Trafton, "The attentional costs of interrupting task performance at various stages," in Human Factors and Ergonomics Society Annual Meeting Proceedings, 2002, pp. 1824-1828.

[23] E. Cutrell, M. Czerwinski, and E. Horvitz, "Notification disruption and memory: Effects of messaging interruptions on memory and performance," in Proceedings of Interact 2001, 2001, pp. 263-269.

[24] F. D. Davis, "Perceived usefulness, perceived ease of use, and user acceptance of information technology," MIS Quarterly, vol. 13, no. 3, pp. 319-340, 1989.

[25] S. Moran, "User Perceptions of System Attributes in Ubiquitous Monitoring: Toward a Model of Behavioural Intention," University of Reading, Reading, 2011. 